\title{
Association between Homocysteine-Related Dietary Patterns and Gestational Diabetes Mellitus: A Study Using the Reduced Rank Regression Method
}

\author{
Hui $W^{1}$, Yu-Hong $L^{3}$, Ling-Peng $L^{2}$, Min-Hui $Y^{3}$, \\ Siyu $\mathbf{W}^{1}$, Gu-Qin $\mathbf{L}^{1}$ and Chun-Yan $\mathrm{S}^{1 *}$ \\ ${ }^{1}$ Department of Nutrition, Seventh People's Hospital of \\ Shanghai University of Traditional Chinese Medicine, \\ Shanghai, China \\ ${ }^{2}$ Department of Clinical Lab, Seventh People's Hospital \\ of Shanghai University of Traditional Chinese Medicine, \\ Shanghai, China \\ ${ }^{3}$ Department of Obstetrics and Gynecology, Seventh \\ People's Hospital of Shanghai University of Traditional \\ Chinese Medicine, Shanghai, China \\ *Corresponding author: Shen Chun-Yan, Department \\ of Nutrition, Seventh People's Hospital of Shanghai \\ University of Traditional Chinese Medicine, Shanghai \\ 200137, China
}

Received: July 31, 2021; Accepted: August 14, 2021; Published: August 21, 2021

\begin{abstract}
Background: This study aimed to evaluate the association between homocysteine-related dietary patterns and gestational diabetes mellitus.

Methods: A total of 488 pregnant women at $24-28$ weeks of gestation between January 2019 and December 2020 were included. Demographic characteristics, dietary intake, and multivitamin supplement intake information were collected using a food frequency questionnaire (FFQ); fasting venous blood samples were collected for serum index detection. Serum homocysteine (Hcy), folic acid, and B12 were selected as response variables, and hyperhomocysteinemia (hHcy)related dietary patterns were extracted using the descending rank regression method. The relationship between the score of hHcy-related dietary patterns and GDM was analyzed using a multivariate logistic regression model.
\end{abstract}

Results: Three hHcy-related dietary patterns were extracted: (mode 1) more meat, cattle meat intake, green leafy vegetables, dark vegetables and soy, and less consumption of shrimp. (mode 2) livestock meat, eggs and more grains, green leafy vegetables, bacteria, algae, dairy, and less nuts intake; and (model 3) livestock meat intake, and less soy intake. Because the explanatory variation of mode 3 was relatively small, it was not retained. Only mode 2 had a positive and significant relationship with the risk of developing GDM. After adjusting for confounding factors, the risk of GDM was significantly increased in the highest quartile array (OR=2.96, 95\% Confidence Interval: 0.939-9.356, $\mathrm{P}=0.004)$. There was no significant correlation between dietary pattern 1 and GDM risk $(P>0.05)$.

Conclusion: Homocysteine-related dietary patterns were positively associated with gestational diabetes mellitus.

Keywords: Reduced rank regression method; Homocysteine; Dietary patterns; Gestational diabetes mellitus

\section{Abbreviations}

GDM: Gestational Diabetes Mellitus; hHcy: Hyperhomocysteinemia; Hcy: Homocysteine; DI: Dietary Indices; PCA: Principal Component Analysis; CA: Cluster Analysis; FA: Factor Analysis; RRR: Reduced Rank Regression; PLS: Partial LeastSquares Regression; BMI: Body Mass Index; FFQ: Food Frequency Questionnaire; OGTT: Oral Glucose Tolerance Test; FBG: Fasting Blood Glucose; 1h PG: 1-h Postprandial Blood Glucose; 2h PG: 2-h Postprandial Blood Glucose

\section{Introduction}

Due to the increase in the incidence of obesity and elderly parturients, the incidence of Gestational Diabetes Mellitus (GDM) in China has been increasing by $14.8 \%$ annually; with regional variation, wherein: East China, Central China, and North China have a higher prevalence [1]. The prognosis of GDM mainly depends on early prevention and intervention, among which dietary therapy is an important strategy for its primary prevention and is the basis of all diabetes treatments [2]. Dietary pattern is a comprehensive evaluation of diet as a whole, which can accurately reflect the effect diet disease [3] and in the relationship between nutrition and health [4]. Studies have found that high homocysteine levels (hyperhomocysteinemia, hHcy) are risk factors for GDM [5], and dietary patterns affect serum homocysteine (Hcy) levels; for example, a mediterranean diet [6] and frugal diet [7] can significantly reduce serum Hcy levels. However, whether dietary patterns affect the incidence of GDM through changes in Hcy levels and its related mechanisms remains elucidated.

There are three types of dietary pattern-extraction methods. First, is the use of dietary indices (DI); second is induction, which includes Principal Component Analysis (PCA), Cluster Analysis (CA), and factor analysis (FA); and third is the combination of methods from the first and second classes, Reduced Rank Regression (RRR) and Partial Least-Squares regression (PLS). Adopting different methods according to the research purpose, the RRR method can explain the reaction variables to the greatest extent, such as variables related to disease outcomes, and nutrients, explain the variations [8], contribute to the analysis of the relationship between dietary patterns and disease, and analyze its possible mechanism. The applications of RRR 
are expanding in the field of nutrition epidemiology [9]. Therefore, this study aimed to extract hHcy-related dietary patterns using the RRR method and analyze their relationship with the incidence of GDM to explore the possible role of dietary patterns in hcy level and GDM.

\section{Methods}

\section{Subjects}

A total of 512 pregnant women at 24-28 weeks of gestation who underwent regular obstetric examinations at our hospital between January 2019 and December 2020 were included in the study. Inclusion criteria were single pregnancy, normal expression and understanding ability, and informed consent. Exclusion criteria were history of diabetes mellitus, hypertension, and thyroid disease; acute or chronic infectious diseases with obvious symptoms; and other major diseases. This study was approved by the Medical Ethics Committee of our hospital. All study participants provided informed consent.

\section{Research methods}

General demographic characteristics: Sociodemographic data (age, education and gestational age) and pregnancy history (number of pregnancies/births) were collected by trained investigators. Height and weight were measured using uniform standards and specifications, and Body Mass Index (BMI) before pregnancy was calculated to record weight gain during pregnancy.

Dietary questionnaire survey: The Food Frequency Questionnaire (FFQ) used a dietary review method and a food model to collect the dietary intake and multivitamin supplement intake of pregnant women during pregnancy in the form of face-to-face interviews. According to the food classification principles in the Chinese food composition List (sixth edition) [10], food types are classified and sorted into 24 types of food groups. All food intake data were standardized using NutriStar software (Yingkang Technology Company). The specific method was as follows: the intake of each food/group was equal to each intake multiplied by the daily intake times. The daily intake times were converted from the intake frequency. Food with an intake proportion $\leq 5 \%$ (crab/shell, egg tarts/ shaomai, coffee/tea, and condiments) were not included in the dietary pattern analysis. In addition, subjects whose intake frequency of the 24 food groups was $>99 \%$ with an energy intake of $\leq 800 \mathrm{kcal}$ were excluded. Finally, 488 cases were included in the analysis.

Diagnostic criteria for gestational diabetes: Pregnant women were screened for gestational diabetes mellitus at 24-28 weeks of gestation (referred to as "glucose screening). Glucose screening was a 75g oral glucose tolerance test (OGTT), China's Guidelines for the Diagnosis and Treatment of Gestational Diabetes Mellitus (2014) [11] GDM is diagnosed if the blood glucose level reaches or exceeds the lower limit as follows: Fasting Blood Glucose (FBG) 5.1mmol/L, 1-h postprandial blood glucose (1-h PG) $10.0 \mathrm{mmol} / \mathrm{L}$, and $2-\mathrm{h}$ postprandial blood glucose (2h PG) $8.5 \mathrm{mmol} / \mathrm{L}$. According to the results from the OGTT, patients were divided into normal and GDM groups.

Serum index detection: The blood glucose in the OGTT was measured using the hexokinase method with a Beckman automatic biochemical analyzer (AU5800). Serum Hcy was detected using the enzyme cycle method with a Beckman automatic biochemical analyzer (AU5811). Serum Folic Acid (FA) and B12 folic acid were determined by the chemiluminescence method using an Abbott Automatic Immunoanalyzer (I1000S). Quality control was performed for all tests prior to testing. When the quality was not controlled, the specific reasons were analyzed and dealt with accordingly. Finally, tests were performed after re-controlling.

\section{Statistical methods}

Differences between the GDM and normal groups were compared using the t-test or $\chi^{2}$ test. RRR analysis was performed with the option (METHOD=RRR) in the PLS process of SAS software version 9.4 (SAS Institute, North Carolina, USA). Serum Hcy, FA, and B12 values were used as response variables and as the RRR method could obtain at most the same number of dietary patterns as the number of response variables, three dietary patterns explaining hHcy variation could be obtained in this study. The dietary pattern factor load represented the size and direction of each food group's contribution to hHcy-related dietary patterns, and the dietary pattern score was obtained by multiplying the dietary pattern factor load by the standardized food intake. The relationship between the scores of the three dietary patterns and the intake of each food group was evaluated using Pearson's correlation. The subjects were divided into four groups according to the quartile of dietary pattern score, the characteristics of the subjects were analyzed, and a trend analysis was performed. The quartiles of dietary scores were used as independent variables, and logistic regression was used to analyze the relationship between hHcy-related dietary pattern scores and GDM after adjusting for age, educational background, gestational grade, BMI before pregnancy, weight gain during pregnancy, energy intake, and multivitamin intake.

\section{Results}

\section{General features}

There was no difference in educational background and weight gain during pregnancy in the GDM group compared to the normal group $(\mathrm{P}<0.01)$. However, patients in the GDM group were older, the proportion of postpartum women was higher, and the pre-pregnancy BMI and energy intake levels were higher $(\mathrm{P}<0.01)$. The intake of folate in the GDM group was lower than that in the normal group, but there was no difference in the intake of $\mathrm{B} 12$ and $\mathrm{B} 6$ between the two groups $(\mathrm{P}>0.01)$. Serum Hcy levels were higher in the GDM group, but FA and B12 levels were lower in the GDM group than in the normal group $(\mathrm{P}<0.01)$ (Table 1$)$.

\section{Characteristics of RRR dietary pattern}

Three dietary patterns were identified in the present study. For mode 1 , the correlation index was $>0.20$ mainly for poultry meat and livestock meat; and $<-0.20$ for green leafy vegetables, dark vegetables, soybeans, and shrimp, which explained the $29.14 \%$ variation in food and $24.26 \%$ variation in response variables. For mode 2 , the correlation index was $>0.20$ mainly for noodles and products, meat and eggs; <-0.20 mainly for grains, green leafy vegetables, bacteria and algae, milk and nuts, which explained the $65.23 \%$ variation in food and $56.38 \%$ variation in response variables. The correlation index of mode 3 factors was $>0.20$ mainly for meat; $<-0.20$ for 
Table 1: Comparison of basic characteristics between the GDM and normal groups.

\begin{tabular}{|c|c|c|c|c|}
\hline \multirow{2}{*}{ Essential Characteristics } & \multicolumn{2}{|c|}{ OGTT } & \multirow{2}{*}{$t$-value } & \multirow{2}{*}{$P$-value } \\
\hline & Normal Group $(n=345)$ & GDM Group $(n=143)$ & & \\
\hline Year & $28.513 \pm 4.447$ & $30.636 \pm 4.645$ & 4.739 & $<0.001$ \\
\hline Educational Background & & & -0.576 & 0.565 \\
\hline Senior High School and Below & $188(54.50)$ & $82(57.34)$ & & \\
\hline College Degree or Above & $157(45.50)$ & $61(42.66)$ & & \\
\hline Gravidity & & & -2.475 & 0.013 \\
\hline Primipara & $180(52.17)$ & $57(39.86)$ & & \\
\hline Multipara & $165(47.83)$ & $86(60.14)$ & & \\
\hline Pregnancy BMI & $22.097 \pm 3.629$ & $23.992 \pm 4.527$ & 4.45 & $<0.001$ \\
\hline Weight Gain During Pregnancy & $8.774 \pm 4.144$ & $8.794 \pm 4.992$ & 0.045 & 0.964 \\
\hline Energy Intake (kcal/d) & $1464.085 \pm 411.720$ & $1642.900 \pm 636.197$ & 3.103 & 0.002 \\
\hline \multicolumn{5}{|l|}{ Multivitamin Supplement Intake } \\
\hline FA (ug) & $538.936 \pm 572.832$ & $431.846 \pm 445.723$ & 1.998 & 0.028 \\
\hline B12 (ug) & $2.631 \pm 6.828$ & $2.472 \pm 9.833$ & 0.204 & 0.838 \\
\hline $\mathrm{B} 6$ (mg) & $1.974 \pm 2.288$ & $1.772 \pm 2.502$ & 0.862 & 0.389 \\
\hline \multicolumn{5}{|l|}{ Serological Indicator } \\
\hline $\mathrm{FA}(\mathrm{nmol} / \mathrm{L})$ & $21.967 \pm 10.523$ & $18.189 \pm 14.161$ & 2.877 & 0.004 \\
\hline B12 (pmol/L) & $219.294 \pm 110.621$ & $188.632 \pm 99.506$ & 2.997 & 0.003 \\
\hline Hcy (umol/L) & $5.770 \pm 1.668$ & $7.235 \pm 5.007$ & 3.423 & $<0.001$ \\
\hline
\end{tabular}

Note: Categorical variables include educational background and pregnancies, expressed as the number of people (constituent ratio). Continuous variables included age, pre-pregnancy BMI, weight gain during pregnancy, energy intake, intake of multivitamin supplements (FA, B12, and B6), and serological indicators (FA, B12, and $\mathrm{Hcy}$ ), expressed as mean \pm standard deviation, in which energy intake did not include the energy provided by cooking oil intake.

Table 2: The load of each food component in hHcy-related dietary pattern and its correlation with dietary pattern score.

\begin{tabular}{|c|c|c|c|c|c|c|}
\hline \multirow{2}{*}{ Food group } & \multicolumn{2}{|c|}{ Pattern 1} & \multicolumn{2}{|c|}{ Pattern 2} & \multicolumn{2}{|c|}{ Pattern 3} \\
\hline & Factor Loading & Correlation Index & Factor Loading & Correlation Index & Factor Loading & Correlation Index \\
\hline Surface and products & -0.077 & $-0.142^{c}$ & $0.231^{\mathrm{a}}$ & $0.564^{c}$ & 0.015 & 0.057 \\
\hline Grains & -0.023 & -0.048 & $-0.316^{a}$ & $-0.222^{c}$ & $-0.040^{a}$ & -0.048 \\
\hline Green leafy vegetables & $-0.222^{a}$ & $-0.160^{c}$ & $-0.233^{a}$ & $-0.559^{c}$ & 0.040 & 0.014 \\
\hline Dark vegetables & $-0.261^{a}$ & $-0.113^{b}$ & 0.046 & $-0.426^{c}$ & 0.010 & 0.082 \\
\hline Thallophyte & 0.143 & $0.131^{\mathrm{c}}$ & $-0.232^{a}$ & $-0.480^{c}$ & 0.022 & 0.010 \\
\hline High energy fruits & 0.130 & $0.600^{c}$ & -0.056 & $-0.505^{c}$ & -0.021 & 0.059 \\
\hline Poultry meat & $0.311^{\mathrm{a}}$ & $-0.168^{c}$ & 0.087 & $0.470^{c}$ & 0.038 & 0.029 \\
\hline Livestock meat & $0.251^{\mathrm{a}}$ & -0.030 & $0.269^{\mathrm{a}}$ & $0.461^{c}$ & 0.237 & $0.239^{c}$ \\
\hline Fish & 0.160 & $0.244^{c}$ & 0.041 & $-0.386^{c}$ & -0.080 & $0.188^{c}$ \\
\hline Shrimp & -0.242 & 0.070 & 0.062 & $-0.273^{c}$ & 0.022 & 0.005 \\
\hline Eggs & 0.178 & -0.048 & $0.291^{\mathrm{a}}$ & $0.515^{c}$ & 0.066 & 0.065 \\
\hline Milk Group & -0.074 & $-0.256^{c}$ & $-0.247^{a}$ & $0.107^{\mathrm{b}}$ & 0.020 & 0.064 \\
\hline Soybeans & $-0.212^{\mathrm{a}}$ & 0.006 & -0.112 & $-0.432^{c}$ & $-0.281^{a}$ & $-0.614^{c}$ \\
\hline Nuts and fruits & 0.178 & $0.236^{c}$ & $-0.577^{a}$ & $-0.436^{c}$ & 0.059 & $0.088^{c}$ \\
\hline Explain the proportion of variation & Pattern & & Pattern 2 & Patt & rn 3 & Summation \\
\hline Explain Each Food Group & 4.250 & & 9.512 & 0.8 & & 14.583 \\
\hline Explanatory Response Variable & 0.361 & & 0.839 & 0.2 & & 1.488 \\
\hline
\end{tabular}

Note: a Only food groups with absolute factor load $>0.20$ are shown. ${ }^{b} \mathrm{P}<0.05,{ }^{\mathrm{c}} \mathrm{P}<0.01$. 
Table 3: Characteristics of subjects under different quartile scores of hHcy-related dietary patterns.

\begin{tabular}{|c|c|c|c|c|c|c|}
\hline & \multicolumn{3}{|c|}{ Pattern 1} & \multicolumn{3}{|c|}{ Pattern 2} \\
\hline & Q1 & Q4 & $P$ values & Q1 & Q4 & $P$ values \\
\hline Year & $29.254 \pm 4.203$ & $28.869 \pm 4.722$ & 0.502 & $28.746 \pm 4.512$ & $30.508 \pm 4.679$ & 0.0046 \\
\hline Educational Background & & & 0.051 & & & 0.054 \\
\hline Senior High School and Below & $77(63.64)$ & $61(50.41)$ & & $54(44.63)$ & $69(57.02)$ & \\
\hline College Degree or Above & $44(36.36)$ & $60(49.59)$ & & $67(55.37)$ & $52(42.98)$ & \\
\hline Gravidity & & & 0.062 & & & 0.072 \\
\hline Primipara & $57(47.11)$ & $72(59.50)$ & & $70(57.85)$ & $55(45.45)$ & \\
\hline Multipara & $64(52.89)$ & $49(40.50)$ & & $51(42.15)$ & $66(54.55)$ & \\
\hline Pregnancy BMI & $22.978 \pm 4.088$ & $22.078 \pm 3.393$ & 0.063 & $21.836 \pm 3.488$ & $23.804 \pm 4.355$ & $<0.001$ \\
\hline Weight gain during pregnancy & $8.863 \pm 4.782$ & $9.502 \pm 4.337$ & 0.276 & $7.947 \pm 4.082$ & $8.930 \pm 5.365$ & 0.1084 \\
\hline \multicolumn{7}{|l|}{ Multivitamin Supplement Intake } \\
\hline FA (ug) & $516.10 \pm 637.70$ & $477.20 \pm 411.10$ & 0.574 & $518.90 \pm 537.60$ & $498.90 \pm 557.90$ & 0.778 \\
\hline B12 (ug) & $2.086 \pm 3.370$ & $2.952 \pm 10.550$ & 0.394 & $2.153 \pm 2.917$ & $2.210 \pm 2.980$ & 0.882 \\
\hline B6 (mg) & $1.770 \pm 2.070$ & $2.010 \pm 2.636$ & 0.433 & $1.830 \pm 1.811$ & $1.760 \pm 1.866$ & 0.768 \\
\hline \multicolumn{7}{|l|}{ Serological Indicator } \\
\hline FA (nmol/L) & $183.6 \pm 110.900$ & $219.5 \pm 82.669$ & 0.0008 & $238.7 \pm 96.952$ & $115.7 \pm 62.542$ & $<0.001$ \\
\hline B12 (pmol/L) & $17.752 \pm 11.976$ & $22.443 \pm 9.465$ & 0.0046 & $23.883 \pm 10.075$ & $9.266 \pm 6.041$ & $<0.001$ \\
\hline Hcy (umol/L) & $7.344 \pm 3.141$ & $5.403 \pm 1.805$ & $<0.001$ & $5.286 \pm 1.337$ & $9.577 \pm 2.833$ & $<0.001$ \\
\hline
\end{tabular}

Note: Categorical variables include educational background and pregnancies, expressed as the number of people (constituent ratio). Continuous variables included age, pre-pregnancy BMI, weight gain during pregnancy, energy intake, intake of multivitamin supplements (FA, B12, and B6), and serological indicators (FA, B12, and Hcy), which were expressed as mean \pm standard deviation.

Table 4: Logistic regression results of the relationship between hHcy-related dietary pattern score and the prevalence of GDM.

\begin{tabular}{|c|c|c|c|c|}
\hline \multirow{2}{*}{ Dietary Pattern Score Quartile } & \multicolumn{2}{|c|}{ Pattern 1} & \multicolumn{2}{|c|}{ Pattern 2} \\
\hline & Model 1 & Model 2 & Model 1 & Model 2 \\
\hline Q1 & 1 & 1 & 1 & 1 \\
\hline Q2 & $0.890(0.461-1.718)$ & $0.809(0.409-1.600)$ & $1.894(0.916-3.917)$ & $1.937(0.911-4.117)$ \\
\hline Q3 & $0.578(0.259-1.290)$ & $0.529(0.230-1.213)$ & $1.983(0.808-4.867)$ & $1.983(0.808-4.867)$ \\
\hline Q4 & $0.518(0.152-1.771)$ & $0.480(0.137-1.684)$ & $2.077(0.812-5.315)^{a}$ & $2.963(0.939-9.356)^{\mathrm{a}}$ \\
\hline$P$ values & 0.35 & 0.45 & 0.005 & 0.004 \\
\hline
\end{tabular}

Note: Model 1: Age, educational background, number of pregnancies, pre-pregnancy BMI, and pregnancy weight gain were adjusted. Model 2: Energy intake and multivitamin supplement intake (folic acid, B12, and B6) were further adjusted. aP $<0.01$.

soybeans, which explained the $5.63 \%$ variation in food and $19.35 \%$ variation in response variables. As the explanation variation of mode 3 was relatively small, it was excluded.

\section{Characteristic analysis of dietary pattern quartile}

Compared with the lowest quartile array of pattern 1 , the subjects in the highest quartile array of pattern 1 had higher energy intake, higher serum Hcy, lower serum FA and B12, and both showed a linear trend. Compared with the lowest quartile array, the subjects in the highest quartile array were older, had higher pre-pregnancy BMI, higher serum Hcy, and lower serum FA and B12, with a linear trend, but there was no difference in energy intake and weight gain during pregnancy (Table 3).

\section{Correlation analysis between dietary pattern and GDM}

Logistic regression analysis showed that after adjusting for multiple confounding factors, the score of mode 2 was significantly positively correlated with the incidence of GDM $(\mathrm{P}<0.01)$, and the risk of GDM significantly increased relative to the lowest quartile array and the fourth quartile array ( $\mathrm{OR}=2.963,95 \% \mathrm{CI}$ : 0.939-9.356). However, there was no significant relationship between the score of mode 1 and the incidence of GDM $(\mathrm{P}>0.05)$. The risk of developing GDM in the lowest and highest quartiles of the scores was $\mathrm{OR}=0.480$ (95\% CI: 0.137-1.684) (Table 4).

\section{Discussion}

In this study, serum Hcy, FA, and B12 were selected as response variables, and three hHcy-related dietary patterns were extracted using the RRR method. These three dietary patterns could explain the variation in serum Hcy, FA, and B12 to the greatest extent from the perspective of food. Similar proportions of variation in response variables have been explained in other clinical studies using the RRR method $[12,13]$. The variation explained by mode 3 was significantly smaller than that of mode 1 and mode 2 , thus, it was excluded. It was found that the scores of modes 1 and 2 were positively correlated with 
the Hcy level, indicating that these two dietary patterns were closely correlated with the Hcy level; this finding is consistent with that of previous studies. Previous studies have found that Hcy levels are significantly correlated with the risk of GDM, which can significantly increase the risk by $20 \%$ [5]. Hcy is a sulfur-containing amino acid, an important intermediate in the methionine metabolism process, and any defects that lead to key enzymes or cofactors can result in methionine cycle problems that affect serum Hcy levels, and a unit of carbon metabolism-related vitamins, such as vitamin B6, vitamin B12, folic acid, and betaine, which are important coenzymes in the metabolism process. In recent years, an increasing number of studies have suggested that Hcy is closely related to insulin resistance [14], and hHcy should be included in metabolic syndrome [15]. The mechanism is thought to be a result of Hcy being a vascular damaging amino acid that can induce vascular damage and oxidative stress in pancreatic beta cells, leading to disorders of glucose and lipid metabolism [16].

Mode 2 was characterized by a dietary pattern with a higher intake of noodles and products, meat and eggs, and less intake of cereals, green leafy vegetables, bacteria and algae, milk, and nuts. Among the two hHcy-related dietary patterns obtained, only mode 2 showed a positive correlation with the prevalence of GDM. This is consistent with previous studies, that state that the dietary pattern with high intake of fruits and vegetables, grains and roots, and milk, is rich in one-carbon unit metabolic-related vitamins, such as vitamin B6, vitamin B12, folic acid, and betaine, which can reduce the blood Hcy level [7]. However, insufficient intake can increase Hcy levels [17]. There are two components of folic acid intake during pregnancy: from multivitamins and from food (animal liver, poultry, eggs, beans, and leafy greens). In China, continuous supplementation of folic acid in the first 3 months of pregnancy and during pregnancy to prevent fetal neural tube defects is a major public health project [18] to ensure successful birth and good childcare [19]. This study found that only $23.30 \%$ of the patients started taking folic acid 3 months before pregnancy, $76.70 \%$ of the patients started taking folic acid after being pregnant, and $77.13 \%$ continued to take folic acid or multivitamins in the second trimester. The results showed that the awareness of taking folic acid supplements during the perinatal period in the Shanghai Gaoqiao area is relatively low, and nutritional education for women of childbearing age needs to be strengthened.

Pattern 1 was characterized by a higher intake of poultry and livestock meat and a lower intake of green leafy vegetables, dark vegetables, beans, and shrimp. Poultry meat, animal meat, and other protein foods are rich in methionine, and a high intake of poultry meat or lack of a carbon unit metabolism-related vitamin will lead to an increase in serum Hcy concentration, which is consistent with previous studies [17]. A prospective clinical study of 681 patients found a significant correlation between meat dietary patterns and the prevalence of GDM [20]. However, this study did not find a significant correlation between dietary pattern score and the prevalence of GDM; this may be because poultry meat offset the risk of GDM caused by elevated serum Hcy levels in other ways. This may be due to the abundance of choline in poultry meat and livestock meat [21], which is another metabolic pathway of Hcy. It can be determined from the one-carbon unit metabolic pathway table that Hcy produces methionine via two methylation pathways: the folate-dependent pathway and the choline/betaine-dependent pathway [22]. The folate-dependent pathway is well known for supplying methyl, while the choline/betaine-dependent pathway has received little attention. Just as a deficiency of folic acid impedes Hcy methylation, individuals who lack choline also have a reduced ability to methylate Hcy, resulting in hHcy [23]. Choline has been suggested as a candidate nutrient intervention for deficient folate intake or metabolic abnormalities [24,25].

The advantage of this study is that two types of hHcy-related dietary patterns were extracted by the RRR method, which explained the variation in $\mathrm{hHcy}$ to the greatest extent, rather than the variation in food. Therefore, if dietary guidance of the population is needed, dietary patterns should be extracted using principal component analysis and other methods. In addition, the relationship between hHcy-related dietary patterns and the incidence of GDM was analyzed, which is of great significance in exploring the relationship between dietary patterns and GDM through Hcy levels. The limitation of this study is that the subjects were from an obstetric clinic of only one hospital, and the sample size was small. In addition, dietary surveys may have a recall bias. As a cross-sectional study, the causal relationship between dietary patterns and GDM could not be determined. In conclusion, hHcy-related dietary pattern scores are significantly correlated with the risk of GDM, and the influence of Hcy level by adjusting dietary pattern to increase food intake rich in one carbon unit metabolismrelated vitamin may contribute to the intervention and prevention of GDM.

\section{Declarations}

Funding: University-level project of Shanghai University of Chinese Medicine (2019LK042).

Ethics Approval: This study was approved by the Medical Ethics Committee of the Seventh People's Hospital Affiliated to Shanghai University of Chinese Medicine (ethics batch number: 2019-7 th $^{\text {- }}$ HIRB-014). All the study participants provided informed consent.

\section{References}

1. Gao C, Sun X, Lu L, Liu F, et al. Prevalence of gestational diabetes mellitus in mainland China: A systematic review and meta-analysis. J Diabetes Investig. 2019; 10: 154-162.

2. Health industry standard of the People's Republic of China. WS331-2011 Diagnosis of gestational diabetes mellitus. Chin J Perinat Med. 2012; 15: 100-106.

3. Zhao-xue Y, Wen-hua Z. Dietary patterns are key to nutrition and health. Chin J Health Manag. 2017; 11: 3-6.

4. Xi P, Liu RH. Whole food approach for type 2 diabetes prevention. Mol Nutr Food Res. 2016; 60: 1819-1836.

5. Gong $\mathrm{T}$, Wang J, Yang M, et al. Serum homocysteine level and gestational diabetes mellitus: A meta-analysis. J Diabetes Investig. 2016; 7: 622-628.

6. Kai-qi L, Xin-yue D, Wen-hua Z. Effects of dietary patterns on human immune function. Chin Med J (Engl). 2020; 100: 3890-3896.

7. Teixeira JA, Steluti J, Gorgulho BM, et al. Prudent dietary pattern influences homocysteine level more than folate, vitamin B12, and docosahexaenoic acid: a structural equation model approach. Eur J Nutr. 2020; 59: 81-91.

8. Ying X. Statistical analysis of dietary patterns and its application. Mod Prev Med. 2013; 40: 1805-1813.

9. Weikert C, Schulze MB. Evaluating dietary patterns: the role of reduced rank regression. Curr Opin Clin Nutr Metab Care. 2016; 19: 341-346. 
10. Yue-xin Y. Chinese Food Ingredients Standard Edition, sixth edition. Peking University Medical Press. 2018.

11. Division of Obstetrics and Gynecology Society of Chinese Medica Association, Cooperative Group of Pregnancy and Diabetes, Chinese Society of Perinatology. Guidelines for the diagnosis and treatment of pregnancy complicated with diabetes. Chin J Obstet Gynecol. 2014; 49: 561-569.

12. Zhao-xue $Y$, Ze-ping $R$, $X u$ Xiao-gang, et al. Association between blood pressure related dietary patterns and identified cognitive performance in the elderly Chinese-a study by reduced rank regression method. Chin J Epidemiology. 2018; 39: 781-785.

13. Mao-wei C, Hui-jun W, Wang Zhi-hong, et al. Study on the correlation between dietary patterns and metabolic syndrome based on reduced rank regression analysis. J Nutr. 2017; 39: 121-126.

14. Wu BTF, Dyer RA, King DJ, et al. Early second trimester maternal plasma choline and betaine are related to measures of early cognitive development in term infants. PLOS ONE. 2012; 7: e43448.

15. Hui Z, Xin-li W, Mei-hua P, Han Tong-yan. Research progress on the effect of folic acid, vitamin B12 and homocysteine on insulin resistance and related mechanisms. Chin J Perinat Med. 2015; 18: 598-600.

16. Zeisel $\mathrm{SH}$, et al. Diet-gene interactions underlie metabolic individuality and influence brain development: implications for clinical practice derived from studies on choline metabolism. Ann Nutr Metab. 2012; 60: 19-25.

17. Shao-jie L, Jian-guo $Y$, Jing $L$, et al. The association between blood homocysteine level and Chinese Healthy Eating Index in a suburb population of Shanghai. Chin J Prev Med. 2021; 55: 485-491.
18. Gan-na C, Min J, Huang Wei-wen, et al. The study of periconception folic acid supplementation and its relationship with pregnancy out-come. Chin J Dis Control Prev. 2021; 25: 160-164.

19. Ao J, Hong-guo W, Zhan Yong-le, et al. Cohort study on the impact of nutrient supplement use during pregnancy on anemia in pregnant women. Chin J Dis Control Prev. 2021; 25: 128-132.

20. Zhi-yi S, Ping G, Man-li S, et al. The association between dietary patterns during pregnancy and the risk of gestational diabetes mellitus. Chinese Journal of Nursing. 2018; 053: 668-673.

21. Bei W, Li-wei S, Zhou Li-li, et al. Association between choline intake during pregnancy and choline metabolism in parturients and infants. Chin J Perinat Med. 2017; 20: 790-795.

22. Durand $\mathrm{P}$, Prost M, Loreau N, et al. Impaired homocysteine metabolism and atherothrombotic disease. Lab Invest. 2001; 81: 645-672.

23. da Costa KA, Gaffney CE, Fischer LM, et al. Choline deficiency in mice and humans is associated with increased plasma homocysteine concentration after a methionine load. Am J Clin Nutr. 2005; 81: 440-444.

24. Olthof MR, Verhoef P. Effects of betaine intake on plasma homocysteine concentrations and consequences for health. Curr Drug Metab. 2005; 6: 1522.

25. Ivanov A, Nash-Barboza S, Hinkis S, et al. Genetic variants in phosphatidylethanolamine $\mathrm{N}$-methyltransferase and methylenetetrahydrofolate dehydrogenase influence biomarkers of choline metabolism when folate intake is restricted. J Am Diet Assoc. 2009; 109: 313-318. 